\title{
Effect of Helicobacter pylori Eradication on Long-Term Survival after Distal Gastrectomy for Gastric Cancer
}

Young-II Kim, MD
Soo-Jeong Cho, MD, PhD
Jong Yeul Lee, MD
Chan Gyoo Kim, MD, PhD
Myeong-Cherl Kook, MD, PhD
Keun Won Ryu, MD, PhD
Young-Woo Kim, MD, PhD
Il Ju Choi, MD, PhD

Center for Gastric Cancer, National Cancer Center, Korea
Correspondence: Il Ju Choi, MD, PhD

Center for Gastric Cancer,

National Cancer Center, 323 Ilsan-ro,

Ilsandong-gu, Goyang 10408, Korea

Tel: 82-31-920-1629

Fax: 82-31-920-0069

E-mail: cij1224@hanmail.net

Received July 19, 2015

Accepted October 27, 2015

Published Online November 17, 2015

\begin{abstract}
Purpose
Negative Helicobacter pylori status has been identified as a poor prognostic factor for survival in gastric cancer (GC) patients who underwent surgery. The aim of this study was to examine the effect of $H$. pylori eradication on long-term outcomes after distal gastrectomy for GC.
\end{abstract}

\section{Materials and Methods}

We analyzed the survival of 169 distal GC patients enrolled in a prospective randomized trial evaluating histologic changes of gastric mucosa after $\mathrm{H}$. pylori eradication in the remnant stomach. The outcomes measured were overall survival (OS) and GC recurrence rates.

\section{Results}

The median follow-up duration was 9.4 years. In the modified intention-to-treat analysis including patients who underwent $H$. pylori treatment $(n=87)$ or placebo $(n=82), 5$-year OS rates were $98.9 \%$ in the treatment group and $91.5 \%$ in the placebo group, and KaplanMeier analysis showed no significant difference in OS ( $p=0.957)$ between groups. In multivariate analysis, no difference in overall mortality was observed between groups (adjusted hazard ratio [aHR] for $\mathrm{H}$. pylori treatment, 0.75 ; $\mathrm{p}=0.495$ ) or $\mathrm{H}$. pylori-eradicated status (aHR for positive $H$. pylori status, 1.16; $p=0.715$ ), while old age, male sex, and advanced stage $\geq$ Illa were independent risk factors. Six patients in the treatment group (6.9\%) and seven patients in the placebo group (8.5\%) had GC recurrences, and GC recurrence rates were not different according to $\mathrm{H}$. pylori treatment (5-year GC recurrence rates, $4.6 \%$ in the treatment group vs. $8.5 \%$ in the placebo group; $p=0.652$ ).

\section{Conclusion}

H. pylori eradication for GC patients who underwent distal gastrectomy did not compromise long-term survival after surgery.

\section{Introduction}

Guidelines recommend Helicobacter pylori eradication in patients who undergo distal gastrectomy for treatment of gastric neoplasia [1]. Despite these recommendations, several studies have reported poor long-term outcomes in patients negative for $H$. pylori infection after gastrectomy [2-4]. In these studies, negative $H$. pylori status was an independent poor prognostic factor for overall survival (OS) [2,4], gastric
Key words

Helicobacter pylori, Stomach neoplasms, Gastrectomy, Survival cancer-specific survival [3], and relapse-free survival [2]. In addition, gastric cancers in patients without $H$. pylori infection had poorer clinicopathological features compared with those in patients with $H$. pylori infection, including larger tumor size, more advanced stage, and more frequent lymph node or distant metastases [3-7].

However, $H$. pylori eradication or $H$. pylori-negative status after endoscopic resection for gastric cancer has been associated with a reduced risk for development of metachronous cancers in the remnant stomach $[8,9]$. In addition, H. pylori 
eradication leads to improvement of premalignant conditions, such as mucosal atrophy and intestinal metaplasia, in the remnant stomach after gastrectomy $[10,11]$. Therefore, $H$. pylori eradication may have beneficial effects, including the potential prevention of metachronous cancers after gastrectomy.

In a previous double-blind randomized controlled study, we reported that $H$. pylori eradication improved the histological changes of the remnant stomach mucosa, such as atrophy and intestinal metaplasia, after subtotal gastrectomy for gastric cancer [10]. In the current study, we evaluated longterm outcomes, including survival and cancer recurrence, to determine whether $H$. pylori eradication has a detrimental effect on long-term survival after distal gastrectomy for gastric cancer.

\section{Materials and Methods}

\section{Study population}

We observed patients enrolled in a previous double-blind randomized controlled trial evaluating the effects of $H$. pylori eradication on mucosal atrophy and intestinal metaplasia in the remnant stomach after distal gastrectomy [10]. Detailed methods were described previously. In brief, newly diagnosed gastric cancer patients aged 18 to 70 years were recruited and screened for eligibility at the National Cancer Center, Korea, between August 2003 and May 2006. Eligibility criteria were as follows: distal gastric adenocarcinoma of clinical stage IA, IB, II, or IIIA according to the 6th edition of the International Union Against Cancer/American Joint Committee on Cancer TNM classification system [12], and positive tests for $H$. pylori infection. Informed consent was obtained from all participants. The Institutional Review Board of the National Cancer Center, Korea, approved the study protocol (NCCCTS03-063) and the original trial was registered with ClinicalTrials.gov (NCT01002443) [10].

\section{H. pylori status evaluation}

Both the rapid urea test (RUT) and histology before distal gastrectomy were used for assessment of $H$. pylori infection. On the initial esophagogastroduodenoscopy (EGD), six biopsy specimens were taken from the lesser curvature of the antrum and the lesser and greater curvatures of the corpus for evaluation of $H$. pylori status, mucosal atrophy, and intestinal metaplasia. The collected biopsy specimens were immediately fixed in neutral-buffered $10 \%$ formalin, embedded in paraffin blocks, and stained with hematoxylin-eosin and Wright-Giemsa for histological evaluation. A single pathologist (M.-C.K.) performed pathological evaluation. One additional biopsy specimen was taken from the greater curvature of the corpus for RUT (Proton Dry, Medical Instruments Corporation, Solothurn, Switzerland). Patients confirmed as $H$. pylori infection by both a positive RUT and histological evaluation were enrolled in the study.

\section{Randomization and surgery}

Eligible patients were randomly assigned to the treatment group or the placebo group by computer-generated random number. All investigators and patients were blinded to the randomization results throughout the trial. The treatment group received proton pump inhibitor (PPI)-based standard triple therapy, consisting of rabeprazole $10 \mathrm{mg}$, clarithromycin $500 \mathrm{mg}$, and amoxicillin 1,000 $\mathrm{mg}$ twice daily for 7 days. The placebo group received the same schedule of rabeprazole $10 \mathrm{mg}$ but received a placebo rather than clarithromycin and amoxicillin. These treatments were administered before distal gastrectomy. Then, all patients underwent an open or laparoscopic distal gastrectomy with D1+ or D2 lymph node dissection [13]. The reconstruction methods were Billroth I (gastroduodenostomy) or II (gastrojejunostomy).

\section{Follow-up H. pylori status}

Follow-up EGDs with biopsy, RUT, and urea breath test (UBT) were performed at 3 months after distal gastrectomy for assessment of $H$. pylori status. The biopsy specimens were taken from the lesser and greater curvatures of the corpus, but not the antrum. At the follow-up examination, H. pylori was considered negative if all three tests were negative.

\section{Statistical analysis}

The calculation of study sample size was described in detail previously [10]. In the current study, outcomes were OS, gastric cancer-specific death, and gastric cancer recurrence. OS and gastric cancer-specific survival were determined from the time of distal gastrectomy to death; OS included deaths from any cause, but gastric cancer-specific death included only death from gastric cancer. Gastric cancer recurrence included metachronous cancer in the remnant stomach, lymph node metastasis, and distant organ metastasis occurring after gastrectomy for distal gastrectomy.

Continuous variables were compared using the MannWhitney $U$ test and categorical variables were compared using the chi-square test or Fisher exact test. Follow-up data on deaths and recurrences were obtained until December 2014. The Kaplan-Meier method was used for construction 
of survival curves, and comparisons were performed using the log-rank test. Comparisons of gastric cancer-specific death and gastric cancer recurrence rates were performed using Gray's test because of the possible competing risk of death without recurrence. A multivariate Cox proportional hazards model analysis was performed for overall mortality, and a multivariate Fine-Gray regression model analysis was performed for cancer recurrence with competing risks. In model 1, multivariate analyses were performed according to the allocated groups, and in model 2, analyses were performed according to the $H$. pylori status at 3 months after gastrectomy. Covariates for multivariate analyses were age, sex, and variables having a p-value less than 0.1 in univariate analyses. Analyses were performed using STATA ver. 12.1 (Stata Corp., College Station, TX), except for the Gray's test, which was analyzed using SAS ver. 9.3 (SAS Inc., Cary, NC). A p-value less than 0.05 was considered statistically significant.

\section{Results}

Of the 190 randomized patients, 169 patients who received treatment or placebo were included in the analyses; 87 in the treatment group and 82 in the placebo group. At 3 months after distal gastrectomy, $H$. pylori infection status was evaluated using UBT, histology, and RUT in 167 patients; 67 patients in the treatment group $(81.7 \%, 67 / 86$ patients) and 15 patients in the placebo group $(18.3 \%, 15 / 81$ patients $)$ were negative for $H$. pylori infection (Fig. 1). These patients were included in the analyses according to the H. pylori status at 3 months after distal gastrectomy.

Baseline characteristics of the 169 allocated patients and 167 patients who underwent follow-up $H$. pylori tests at 3 months after gastrectomy are summarized in Table 1 . Median age was 57 years (interquartile range [IQR], 48 to 64 years) and the proportion of male patients was $69.2 \%$ (117 patients). There were no differences in age, sex, smoking, alcohol drinking, surgical methods, reconstruction methods, pathologic stage, and histology between the treatment and placebo groups. Comparisons according to $H$. pylori status at 3 months after gastrectomy showed no significant differences in baseline characteristics except for the final cancer stage after surgery; a higher proportion of patients with $H$. pyloripositive status had stage I cancer compared to those with H. pylori-negative status $(80.0 \%$ vs. $72.0 \%$, respectively; $\mathrm{p}=0.033$ ).

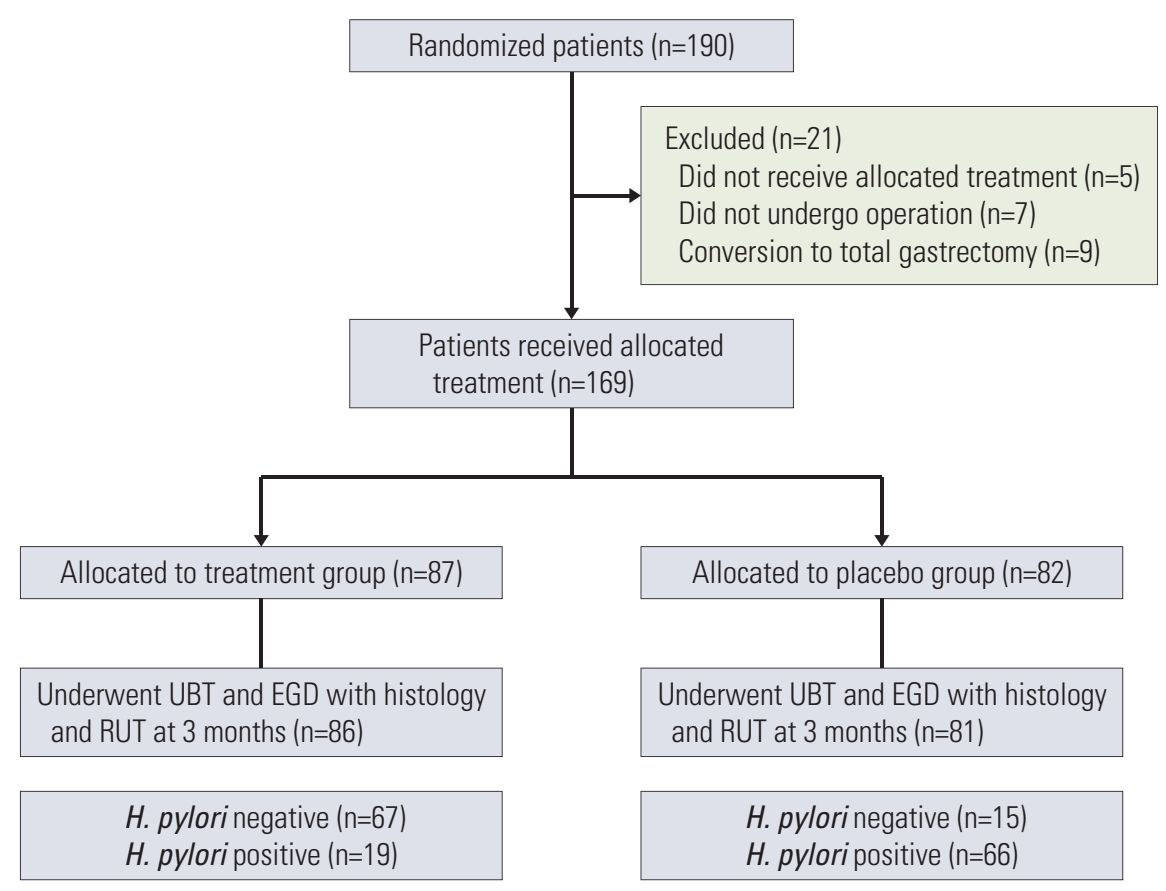

Fig. 1. Study flowchart. EGD, esophagogastroduodenoscopy; H. pylori, Helicobacter pylori; RUT, rapid urease test; UBT, urea breath test. 
Table 1. Baseline characteristics

\begin{tabular}{|c|c|c|c|c|c|c|}
\hline \multirow{2}{*}{ Characteristic } & \multicolumn{3}{|c|}{ Initial allocation group } & \multicolumn{3}{|c|}{$\begin{array}{l}\text { Helicobacter pylori status at } \\
3 \text { months after surgery }\end{array}$} \\
\hline & $\begin{array}{l}\text { Placebo } \\
(n=82)\end{array}$ & $\begin{array}{l}\text { Treatment } \\
(\mathrm{n}=87)\end{array}$ & p-value & $\begin{array}{l}\text { Negative } \\
(n=82)\end{array}$ & $\begin{array}{l}\text { Positive } \\
(n=85)\end{array}$ & p-value \\
\hline Age, median (IQR, yr) & $56(48-64)$ & $58(48-65)$ & 0.715 & $57(46-64)$ & $58(48-64)$ & 0.743 \\
\hline \multicolumn{7}{|l|}{ Sex } \\
\hline Female & $21(25.6)$ & $31(35.6)$ & 0.158 & $25(30.5)$ & $27(31.8)$ & 0.859 \\
\hline Male & $61(74.4)$ & $56(64.4)$ & & $57(69.5)$ & $58(68.2)$ & \\
\hline \multicolumn{7}{|l|}{ Allocation group } \\
\hline Placebo & - & - & - & $15(18.3)$ & $66(77.7)$ & $<0.001$ \\
\hline Treatment & - & - & & $67(81.7)$ & $19(22.4)$ & \\
\hline \multicolumn{7}{|l|}{ Alcohol drinking } \\
\hline No & $35(42.7)$ & $40(46.0)$ & 0.667 & $38(46.3)$ & $36(42.4)$ & 0.604 \\
\hline Yes & $47(57.3)$ & $47(54.0)$ & & $44(53.7)$ & $49(57.7)$ & \\
\hline \multicolumn{7}{|l|}{ Smoking } \\
\hline No & $51(62.2)$ & $46(52.9)$ & 0.221 & 47 (57.3) & $49(57.7)$ & 0.996 \\
\hline Yes & $31(37.8)$ & $41(47.1)$ & & $35(42.7)$ & $36(42.4)$ & \\
\hline \multicolumn{7}{|l|}{ Surgical method } \\
\hline Laparoscopic & $21(25.6)$ & $17(19.5)$ & 0.345 & $15(18.3)$ & $22(25.9)$ & 0.238 \\
\hline Open & $61(74.4)$ & $70(80.5)$ & & $67(81.7)$ & $63(74.1)$ & \\
\hline \multicolumn{7}{|l|}{ Reconstruction method } \\
\hline Billroth I (gastroduodenostomy) & $63(76.8)$ & $59(67.8)$ & 0.191 & $59(72.0)$ & $61(71.8)$ & 0.979 \\
\hline Billroth II (gastrojejunostomy) & $19(23.2)$ & $28(32.2)$ & & $23(28.1)$ & $24(28.2)$ & \\
\hline \multicolumn{7}{|l|}{ Histologic type } \\
\hline Differentiated & $48(58.5)$ & $51(58.6)$ & 0.991 & $45(54.9)$ & $54(63.5)$ & 0.255 \\
\hline Undifferentiated & $34(41.5)$ & $36(41.4)$ & & $37(45.1)$ & $31(36.5)$ & \\
\hline \multicolumn{7}{|l|}{ Final cancer stage after surgery } \\
\hline Stage I & $66(80.5)$ & $63(72.4)$ & 0.257 & $59(72.0)$ & $68(80.0)$ & 0.033 \\
\hline Stage II & $10(12.2)$ & $19(21.8)$ & & $20(24.4)$ & $9(10.6)$ & \\
\hline Stage IIIa or more & $6(7.3)$ & $5(5.8)$ & & $3(3.7)$ & $8(9.4)$ & \\
\hline
\end{tabular}

Values are presented as number $(\%)$ unless otherwise indicated. IQR, interquartile range.

\section{Comparisons of long-term survival rates after distal gas- trectomy}

During a median follow-up period of 9.4 years (IQR, 9.0 to 10.2 years; range, 0.7 to 11.3 years), 26 patients died: 14 in the treatment group and 12 in the placebo group. The 5-year OS rates were $98.9 \%$ in the treatment group and $91.5 \%$ in the placebo group, and results of Kaplan-Meier analysis showed no significant difference between groups ( $p=0.957$ by logrank test) (Fig. 2A). Overall, 13 patients had gastric cancer recurrence (6 [6.9\%] in the treatment group and 7 [8.5\%] in the placebo group). Of these, metachronous cancers occurred in 4 patients ( 3 patients in the treatment group and 1 patient in the placebo group), and the metachronous cancer rates were not different between the treatment group and the placebo group $(3.5 \%$ vs. $1.5 \%, \mathrm{p}=0.341)$. One patient died of gastric cancer metastasis at 5 months after total gastrectomy for metachronous cancer, while, the remaining nine patients had lymph node or other organ metastases ( 3 patients in the treatment group and 6 patients in the placebo group). Of the patients with gastric cancer recurrence, 10 patients died because of gastric cancer recurrence (4 patients in the treatment group and 6 patients in the placebo group). The 5-year incidence rates of gastric cancer recurrence were $4.6 \%$ in the treatment group and $8.5 \%$ in the placebo group, and Gray's test showed no statistical significance $(\mathrm{p}=0.652)$ (Fig. 2B). No difference in the cumulative incidences of gastric cancerspecific death were observed between the treatment and placebo groups (5-year cumulative incidence rate, $1.1 \%$ vs. $6.1 \%$, respectively; $\mathrm{p}=0.422$ by Gray's test) (Fig. 2C).

At 3 months after gastrectomy, 82 patients had negative $H$. pylori status and 85 had positive H. pylori status. After 
A
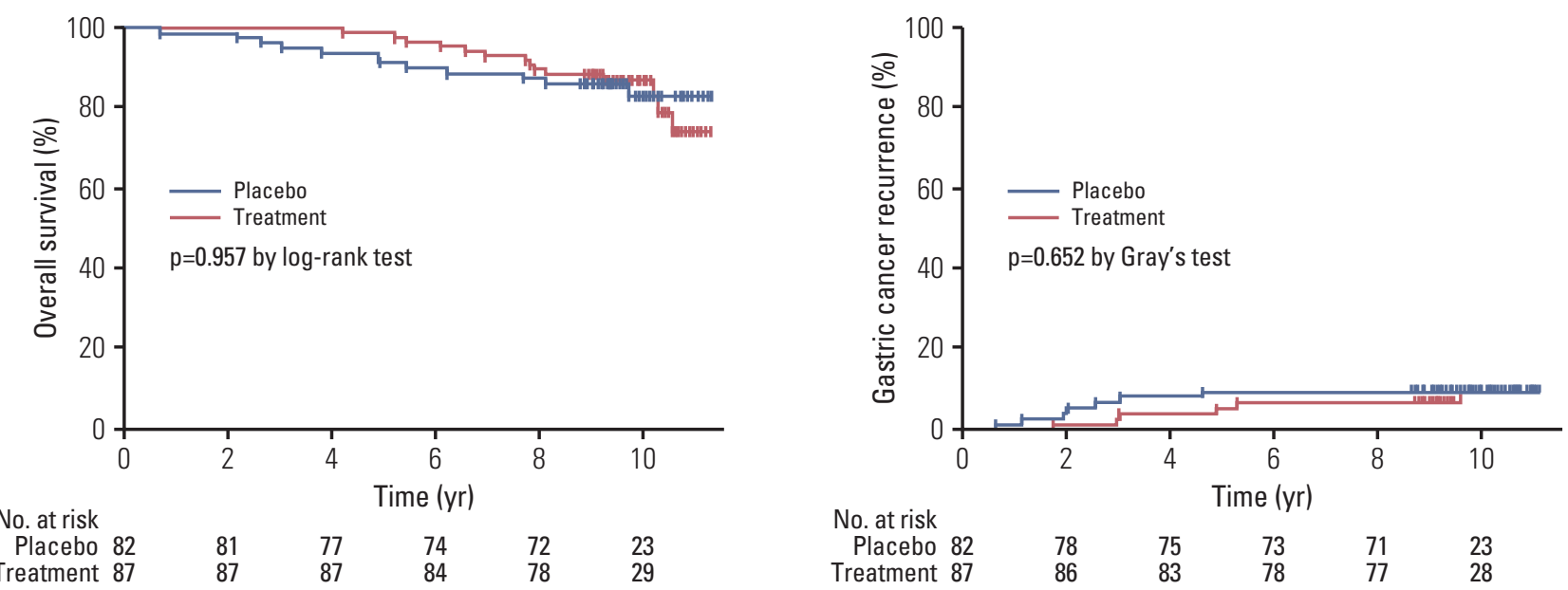

C

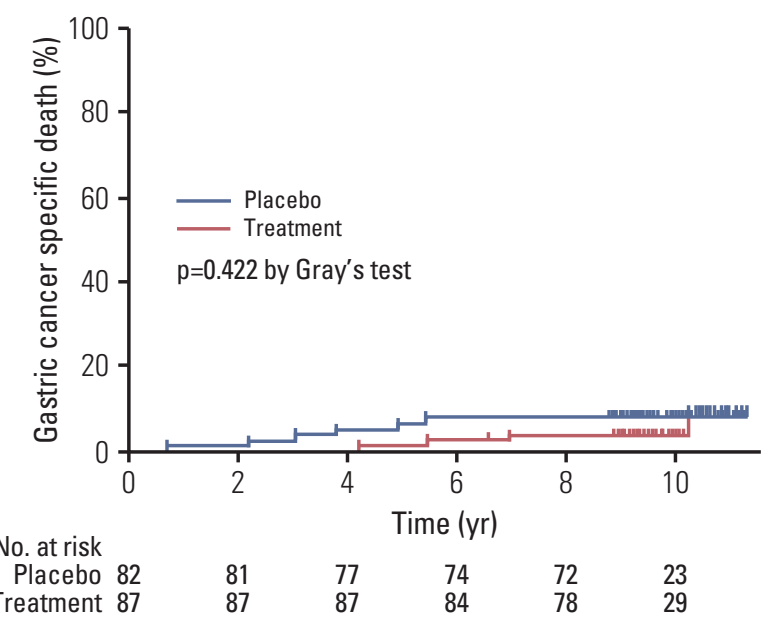

Fig. 2. Comparisons of long-term outcomes according to Helicobacter pylori treatment allocation. Overall survival (A), gastric cancer recurrence (B), and gastric cancer specific death curves (C).

gastrectomy, OS did not differ between patients with negative H. pylori status and those with positive H. pylori status (5-year OS rates, $96.3 \%$ vs. $94.1 \%$, respectively; $\mathrm{p}=0.997$ by log-rank test) (Fig. 3A). In addition, no statistical differences in gastric cancer recurrence (5-year cumulative incidence rates, $6.1 \%$ for negative status vs. $7.1 \%$ for positive status; $\mathrm{p}=0.897$ by Gray's test) (Fig. 3B) and gastric cancer-specific death (5-year cumulative incidence rates, 3.7\% for negative status vs. $3.5 \%$ for positive status; $\mathrm{p}=0.792$ by Gray's test) (Fig. 3C) were observed between patients with and without H. pylori at 3 months after distal gastrectomy.

\section{Univariate and multivariate analyses for overall mortal- ity and cancer recurrence}

In univariate analyses, overall mortality after distal gastrectomy was not affected by $H$. pylori treatment allocation (crude hazard ratio [cHR] for $H$. pylori treatment, 1.02; $\mathrm{p}=0.957$ ) or positive $H$. pylori status at 3 months after distal gastrectomy (cHR for H. pylori-positive, 1.00; $\mathrm{p}=0.997$ ). Male sex, Billroth II anastomosis after distal gastrectomy, and stage $\geq$ IIIa were significant factors associated with overall mortality. In model 1, multivariate analysis according to H. pylori treatment allocation showed that age (adjusted HR [aHR], 1.06; $\mathrm{p}=0.017)$, male sex $(\mathrm{aHR}, 8.38 ; \mathrm{p}=0.005)$, and 
A
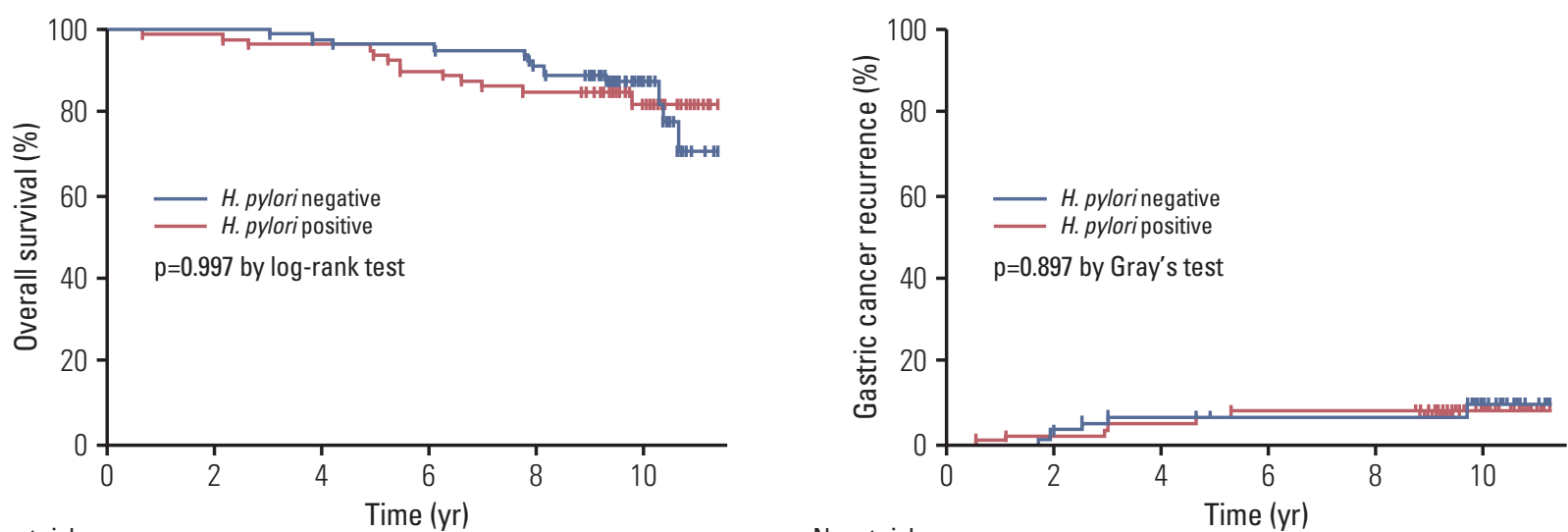

$\begin{array}{cccccc}\text { No. at risk } & & & & \\ \text { H. pylori negative } 82 & 82 & 80 & 79 & 75 & 25 \\ \text { H. pylori positive } 85 & 84 & 82 & 77 & 73 & 26\end{array}$

No. at risk
H. pylori negative 82
H. pylori positive 85

$\begin{array}{lllll}80 & 76 & 74 & 73 & 24 \\ 82 & 80 & 75 & 73 & 26\end{array}$

C

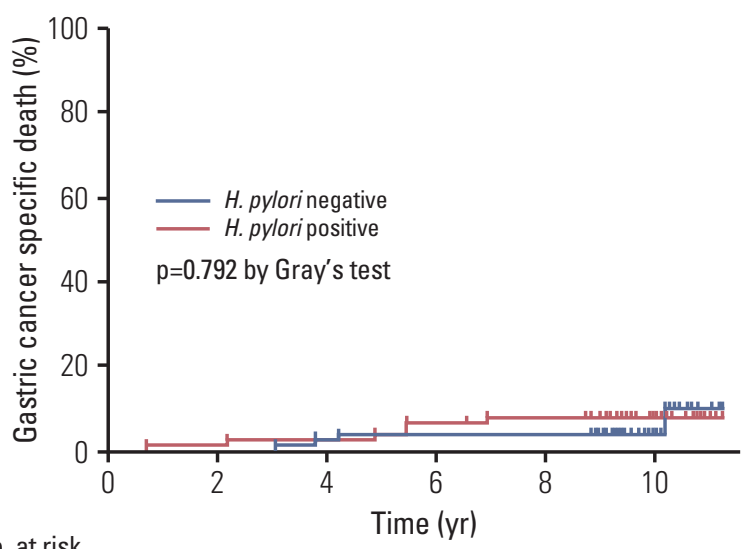

$\begin{array}{rlllll}\text { H. pylori negative } 82 & 82 & 80 & 79 & 75 & 25 \\ \text { H. pylori positive } 85 & 84 & 82 & 77 & 73 & 26\end{array}$

Fig. 3. Comparisons of long-term outcomes according to Helicobacter pylori status at 3 months after distal gastrectomy. Overall survival (A), gastric cancer recurrence (B), and gastric cancer specific death curves (C).

advanced stage $\geq$ IIIa (aHR, 9.18; $\mathrm{p}<0.001$ ) were independent risk factors for overall mortality. H. pylori treatment was not a significant factor for overall mortality (aHR for treatment group, $0.75 ; \mathrm{p}=0.495)$. In model 2 , positive $H$. pylori status at 3 months after gastrectomy did not show significant association with overall mortality (aHR, 1.16; $\mathrm{p}=0.715)$, whereas age, male sex, and advanced stage $\geq$ IIIla were independent risk factors for overall mortality (Table 2).

For cancer recurrence, neither $H$. pylori treatment (cHR, $0.78 ; \mathrm{p}=0.649$ ) nor positive $H$. pylori status after gastrectomy (cHR, 1.13; $\mathrm{p}=0.821$ ) were significant factors in univariate analyses. Multivariate Fine-Gray regression analyses showed that only the advanced stage $\geq$ IIIa was a significant risk fac- tor in both model 1 and 2. In these analyses, H. pylori treatment ( $\mathrm{aHR}, 0.60 ; \mathrm{p}=0.431)$ and positive $H$. pylori status at 3 months after distal gastrectomy (aHR, 1.05; $\mathrm{p}=0.921) \mathrm{did}$ not affect cancer recurrence (Table 3 ).

\section{Discussion}

In the current long-term follow-up study of a double-blind randomized trial, $H$. pylori treatment and $H$. pylori infection status did not affect long-term outcomes after distal gastrec- 
Table 2. Clinical and pathological factors associated with overall mortality

\begin{tabular}{|c|c|c|c|c|c|}
\hline \multirow{2}{*}{ Factor } & \multirow{2}{*}{ No. } & \multicolumn{2}{|c|}{ Univariate analysis $^{\text {a) }}$} & \multicolumn{2}{|c|}{ Multivariate analysis $^{\mathrm{a}}$} \\
\hline & & cHR $(95 \% \mathrm{CI})$ & p-value & aHR $(95 \%$ CI $)$ & p-value \\
\hline \multicolumn{6}{|c|}{ Model 1. Helicobacter pylori allocation group } \\
\hline \multicolumn{6}{|c|}{ Allocation group } \\
\hline Placebo & 82 & 1.00 & & 1.00 & \\
\hline Treatment & 87 & $1.02(0.47-2.21)$ & 0.957 & $0.75(0.32-1.73)$ & 0.495 \\
\hline Age (yr) & 169 & $1.04(0.99-1.08)$ & 0.119 & $1.06(1.01-1.11)$ & 0.017 \\
\hline \multicolumn{6}{|l|}{ Sex } \\
\hline Female & 52 & 1.00 & & 1.00 & \\
\hline Male & 117 & $6.11(1.44-25.87)$ & 0.014 & $8.38(1.87-37.42)$ & 0.005 \\
\hline \multicolumn{6}{|l|}{ Alcohol drinking } \\
\hline No & 75 & 1.00 & & - & \\
\hline Yes & 94 & $1.23(0.56-2.69)$ & 0.612 & - & - \\
\hline \multicolumn{6}{|l|}{ Smoking } \\
\hline No & 97 & 1.00 & & - & \\
\hline Yes & 72 & $1.50(0.69-3.24)$ & 0.305 & - & - \\
\hline \multicolumn{6}{|l|}{ Surgical method } \\
\hline Laparoscopic & 38 & 1.00 & & 1.00 & \\
\hline Open & 131 & $3.77(0.89-15.97)$ & 0.071 & $2.10(0.47-9.43)$ & 0.335 \\
\hline \multicolumn{6}{|l|}{ Reconstruction method } \\
\hline Billroth I (gastroduodenostomy) & 122 & 1.00 & & 1.00 & \\
\hline Billroth II (gastrojejunostomy) & 47 & $3.10(1.42-6.74)$ & 0.004 & $1.56(0.62-3.92)$ & 0.344 \\
\hline \multicolumn{6}{|l|}{ Histologic type } \\
\hline Differentiated & 99 & 1.00 & & - & \\
\hline Undifferentiated & 70 & $0.68(0.30-1.53)$ & 0.348 & - & - \\
\hline \multicolumn{6}{|l|}{ Final cancer stage after surgery } \\
\hline Stage I & 129 & 1.00 & & 1.00 & \\
\hline Stage II & 29 & $1.63(0.58-4.60)$ & 0.355 & $1.80(0.59-5.46)$ & 0.298 \\
\hline Stage IIIa or more & 11 & $10.45(4.31-25.38)$ & $<0.001$ & $9.18(3.25-25.97)$ & $<0.001$ \\
\hline \multicolumn{6}{|c|}{ Model 2. H. pylori status at 3 months after surgery ${ }^{\text {b) }}$} \\
\hline \multicolumn{6}{|c|}{ H. pylori status at 3 months after surgery } \\
\hline Negative & 82 & 1.00 & & 1.00 & \\
\hline Positive & 85 & $1.00(0.46-2.16)$ & 0.997 & $1.16(0.52-2.57)$ & 0.715 \\
\hline
\end{tabular}

cHR, crude hazard ratio; CI, confidence interval; aHR, adjusted hazard ratio. ${ }^{a}$ Cox proportional hazards model was used

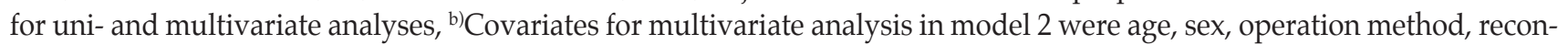
struction method, and final cancer stage after surgery.

tomy, including OS, gastric cancer recurrence, and gastric cancer-specific death. Several studies have reported an association of $H$. pylori infection status with long-term outcomes after gastrectomy. One prospective [2] and two retrospective studies [3,4] reported that negative $H$. pylori status is a poor prognostic factor for long-term outcomes after gastrectomy. Meimarakis et al. [2] reported that negative H. pylori status after surgery is an independent risk factor associated with poor OS and recurrence-free survival, and Marrelli et al. [3] reported that negative $H$. pylori status is a poor prognostic factor for OS. Kang et al. [4] reported an association of negative $H$. pylori status with poor OS in patients who received adjuvant chemotherapy for locally advanced gastric cancer (stage IB to IV) after gastrectomy. In these studies, the negative $H$. pylori status group likely included heterogeneous patients who were initially negative for $H$. pylori infection or those whose $H$. pylori infection disappeared because of the advances of atrophy or $H$. pylori treatment. It appears that negative $H$. pylori status due to any cause may affect longterm survival after gastrectomy. However, our findings suggest that negative $H$. pylori status resulting from $H$. pylori treatment does not affect long-term outcomes after distal gastrectomy.

In comparison with $H$. pylori-positive gastric cancer, 
Table 3. Clinical and pathological factors associated with gastric cancer recurrence

\begin{tabular}{|c|c|c|c|c|c|}
\hline \multirow{2}{*}{ Factor } & \multirow{2}{*}{ No. } & \multicolumn{2}{|c|}{ Univariate analysis $^{\text {a) }}$} & \multicolumn{2}{|c|}{ Multivariate analysis ${ }^{a}$} \\
\hline & & cHR $(95 \%$ CI $)$ & p-value & aHR $(95 \%$ CI $)$ & p-value \\
\hline \multicolumn{6}{|c|}{ Model 1. Helicobacter pylori allocation group } \\
\hline \multicolumn{6}{|c|}{ Allocation group } \\
\hline Placebo & 82 & 1.00 & & 1.00 & \\
\hline Treatment & 87 & $0.78(0.26-2.29)$ & 0.649 & $0.60(0.17-2.12)$ & 0.431 \\
\hline Age (yr) & 169 & $1.03(0.96-1.10)$ & 0.359 & $1.06(0.99-1.13)$ & 0.101 \\
\hline \multicolumn{6}{|l|}{ Sex } \\
\hline Female & 52 & 1.00 & & 1.00 & \\
\hline Male & 117 & $2.50(0.55-11.35)$ & 0.236 & $3.38(0.56-20.54)$ & 0.185 \\
\hline \multicolumn{6}{|l|}{ Alcohol drinking } \\
\hline No & 75 & 1.00 & & - & \\
\hline Yes & 94 & $1.31(0.44-3.96)$ & 0.629 & - & - \\
\hline \multicolumn{6}{|l|}{ Smoking } \\
\hline No & 97 & 1.00 & & - & \\
\hline Yes & 72 & $2.27(0.75-6.85)$ & 0.145 & - & - \\
\hline \multicolumn{6}{|l|}{ Surgical method } \\
\hline Laparoscopic & 38 & 1.00 & & - & \\
\hline Open & 131 & $1.63(0.36-7.33)$ & 0.527 & - & - \\
\hline \multicolumn{6}{|l|}{ Reconstruction method } \\
\hline Billroth I (gastroduodenostomy) & 122 & 1.00 & & 1.00 & \\
\hline Billroth II (gastrojejunostomy) & 47 & $3.22(1.10-9.47)$ & 0.033 & $2.13(0.48-9.40)$ & 0.319 \\
\hline \multicolumn{6}{|l|}{ Histologic type } \\
\hline Differentiated & 99 & 1.00 & & - & \\
\hline Undifferentiated & 70 & $0.61(0.19-2.00)$ & 0.416 & - & - \\
\hline \multicolumn{6}{|l|}{ Final cancer stage after surgery } \\
\hline Stage I & 129 & 1.00 & & 1.00 & \\
\hline Stage II & 29 & $2.30(0.56-9.44)$ & 0.247 & $2.50(0.44-14.03)$ & 0.299 \\
\hline Stage IIIa or more & 11 & $8.76(2.58-29.70)$ & $<0.001$ & $7.44(1.55-35.58)$ & 0.012 \\
\hline \multicolumn{6}{|c|}{ Model 2. H. pylori status at 3 months after surgery ${ }^{\text {b) }}$} \\
\hline \multicolumn{6}{|c|}{ H. pylori status at 3 months after surgery } \\
\hline Negative & 82 & 1.00 & & 1.00 & \\
\hline Positive & 85 & $1.13(0.38-3.35)$ & 0.821 & $1.05(0.37-2.97)$ & 0.921 \\
\hline
\end{tabular}

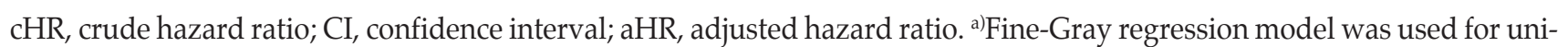
and multivariate analyses, ${ }^{b}$ Covariates for multivariate analysis in model 2 were age, sex, reconstruction method, and final cancer stage after surgery.

H. pylori-negative gastric cancer had the following clinical characteristics: prevalence in younger patients, tumor location in the upper-third portion of the stomach, and more frequent diffuse-type histology $[5,7,14]$. In addition, H. pylori-negative patients had higher advanced cancer stage [5] or distant metastasis [7], suggesting poor clinical outcomes. In the aforementioned studies that reported poorer long-term survival rates after gastrectomy in $H$. pylori-negative gastric cancer patients, baseline clinical characteristics were also different between $H$. pylori-negative and -positive groups. $H$. pylori-negative patients had a significantly higher proportion of tumors located proximally [2,3], and more of these patients underwent total gastrectomy [2]. H. pylori-negative patients had more advanced tumor stage [3,4] and larger tumor size [4] than $H$. pylori-positive patients. These findings suggest that $H$. pylori-negative gastric cancer may be intrinsically associated with poor clinicopathological features. In a recent study, gastric cancers were classified according to molecular subtype [15]. H. pylori infection is also related to some of the molecular changes associated with gastric carcinogenesis, including genetic instability and epigenetic changes in oncogenes or tumor suppressor genes [16]. Different molecular changes are likely in H. pylori-negative gastric cancer compared with $H$. pylori-positive gastric can- 
cer, which may be associated with poor long-term outcomes.

A possible mechanism for the prognostic role of $H$. pylori infection after gastrectomy is that $H$. pylori-induced tumor-specific immune responses may be down-regulated in $H$. pylori-negative gastric cancer patients, possibly influencing long-term outcomes [2]. However, this mechanism has not been proven in clinical studies, and other factors could affect the outcomes for these patients. In the current study, long-term outcomes were not affected by $H$. pylori status after gastrectomy; however, age, male sex, and advanced stage were independent risk factors for overall mortality. Advanced stage was also a significant risk factor for cancer recurrence. In addition, Hobsley et al. [17] commented that negative $H$. pylori status might simply be associated with more advanced tumor progression rather than related to poor prognosis, consistent with our findings.

In our previous study, most $H$. pylori-negative gastric cancers occurred in patients with past $H$. pylori infection, and patients who were truly negative for $H$. pylori infection were rare, occurring at a rate of $2.3 \%$ [7]. Compared with true $H$. pylori-negative gastric cancer patients, gastric cancer patients with past $H$. pylori infection were older and predominantly male, and had a higher proportion of intestinal-type histology and a lower distant metastasis rate [7]. In the current study, H. pylori-negative patients were those with past H. pylori infection that was eradicated upon treatment with PPI-based standard triple therapy. Clinical characteristics of patients in the $H$. pylori-negative group may be different from those of true H. pylori-negative gastric cancer patients, and in this instance, long-term outcomes after distal gastrectomy were not affected by $H$. pylori treatment.

Because of the possible roles of $H$. pylori infection in carcinogenesis in the remnant stomach mucosa after treatment of gastric cancer [18], guidelines recommend H. pylori eradication to prevent development of new cancers in patients who undergo distal gastrectomy [1]. In patients undergoing gastrectomy, $H$. pylori infection may induce chronic and active gastritis, and is associated with increased corpus mucosal cell proliferation in the remnant stomach [18-20]. By contrast, $H$. pylori-negative status is associated with a low risk of mucosal atrophy and intestinal metaplasia in the remnant stomach after gastrectomy $[10,11,18,21]$. In addition, in early gastric cancer patients undergoing endoscopic resection, a previous randomized controlled study reported that H. pylori treatment reduces the incidence of metachronous cancers [8], and a recent meta-analysis also reported significantly reduced incidences of metachronous gastric neoplasia for $H$. pylori-eradicated patients [22]. However, in the current study metachronous cancer rates were not different according to $H$. pylori treatment or according to H. pylori status. This may be because all of the included patients underwent gastric resection of the distal portion of the stomach, which is the common site for synchronous [23] or metachronous cancer [24]. Studies with a proper design and adequate sample size are needed to evaluate the question of whether $H$. pylori eradication may be a preventive strategy for development of metachronous gastric cancer after distal gastrectomy.

The main strength of this study was that it collected longterm follow-up data from gastric cancer patients who underwent distal gastrectomy in a prospective double-blind randomized controlled trial. Thus, we performed intentionto-treat analysis to determine whether $H$. pylori treatment affects long-term outcomes after distal gastrectomy in gastric cancer patients.

However, the current study had several limitations. First, the study sample size may not be large enough to evaluate the association between $H$. pylori infection status and longterm outcomes, because the sample size originally calculated was for the first study, which assessed the histological improvement of the lessor curvature of the corpus after distal gastrectomy [10]. Thus, an additional study including more enrolled patients is needed to provide adequate power. Second, the findings of the current study may not be applicable to areas where the prevalence of $H$. pylori infection and the incidence of gastric cancer are low. Third, spontaneous clearance of $H$. pylori infection during follow-up after gastrectomy was reported in the previous study [25]. Therefore, some $H$. pylori-positive patients in the current study might have spontaneous negative conversion of $H$. pylori infection after distal gastrectomy, and long-term outcomes might be affected by the spontaneous clearance of $H$. pylori infection. Finally, clinicopathological characteristics according to the virulence factors of $H$. pylori strains were not evaluated in the current study.

\section{Conclusion}

In conclusion, H. pylori eradication for gastric cancer patients who undergo distal gastrectomy appears to have no effect on long-term survival and cancer recurrence rates after surgery. Thus, $H$. pylori eradication in gastric cancer patients after distal gastrectomy may be performed without concern for compromising long-term survival.

\section{Conflicts of Interest}

Conflict of interest relevant to this article was not reported. 


\section{Acknowledgments}

This work was supported by grant 1310280 from the National Cancer Center, Korea.

\section{References}

1. Choi IJ. Current evidence of effects of Helicobacter pylori eradication on prevention of gastric cancer. Korean J Intern Med. 2013;28:525-37.

2. Meimarakis G, Winter H, Assmann I, Kopp R, Lehn N, Kist $\mathrm{M}$, et al. Helicobacter pylori as a prognostic indicator after curative resection of gastric carcinoma: a prospective study. Lancet Oncol. 2006;7:211-22.

3. Marrelli D, Pedrazzani C, Berardi A, Corso G, Neri A, Garosi L, et al. Negative Helicobacter pylori status is associated with poor prognosis in patients with gastric cancer. Cancer. 2009; 115:2071-80.

4. Kang SY, Han JH, Ahn MS, Lee HW, Jeong SH, Park JS, et al. Helicobacter pylori infection as an independent prognostic factor for locally advanced gastric cancer patients treated with adjuvant chemotherapy after curative resection. Int J Cancer. 2012;130:948-58.

5. Yoon H, Kim N, Lee HS, Shin CM, Park YS, Lee DH, et al. Helicobacter pylori-negative gastric cancer in South Korea: incidence and clinicopathologic characteristics. Helicobacter. 2011;16:382-8.

6. Qiu HB, Zhang LY, Keshari RP, Wang GQ, Zhou ZW, Xu DZ, et al. Relationship between $\mathrm{H}$. pylori infection and clinicopathological features and prognosis of gastric cancer. BMC Cancer. 2010;10:374.

7. Kwak HW, Choi IJ, Cho SJ, Lee JY, Kim CG, Kook MC, et al. Characteristics of gastric cancer according to Helicobacter pylori infection status. J Gastroenterol Hepatol. 2014;29:1671-7.

8. Fukase K, Kato M, Kikuchi S, Inoue K, Uemura N, Okamoto $\mathrm{S}$, et al. Effect of eradication of Helicobacter pylori on incidence of metachronous gastric carcinoma after endoscopic resection of early gastric cancer: an open-label, randomised controlled trial. Lancet. 2008;372:392-7.

9. Kim YI, Choi IJ, Kook MC, Cho SJ, Lee JY, Kim CG, et al. The association between Helicobacter pylori status and incidence of metachronous gastric cancer after endoscopic resection of early gastric cancer. Helicobacter. 2014;19:194-201.

10. Cho SJ, Choi IJ, Kook MC, Yoon H, Park S, Kim CG, et al. Randomised clinical trial: the effects of Helicobacter pylori eradication on glandular atrophy and intestinal metaplasia after subtotal gastrectomy for gastric cancer. Aliment Pharmacol Ther. 2013;38:477-89.

11. Hwang JJ, Lee DH, Kang KK, Lee AR, Yoon H, Shin CM, et al. Eradication rate and histological changes after Helicobacter pylori eradication treatment in gastric cancer patients following subtotal gastrectomy. World J Gastroenterol. 2015;21: 3936-43.
12. Sobin LH, Wittekind C. TNM classification of malignant tumours. 6th ed. New York: Wiley-Liss; 2002.

13. Japanese Gastric Cancer Association. Japanese gastric cancer treatment guidelines 2010 (ver. 3). Gastric Cancer. 2011;14:113-23.

14. Matsuo T, Ito M, Takata S, Tanaka S, Yoshihara M, Chayama K. Low prevalence of Helicobacter pylori-negative gastric cancer among Japanese. Helicobacter. 2011;16:415-9.

15. Cancer Genome Atlas Research Network. Comprehensive molecular characterization of gastric adenocarcinoma. Nature. 2014;513:202-9.

16. Hanada K, Graham DY. Helicobacter pylori and the molecular pathogenesis of intestinal-type gastric carcinoma. Expert Rev Anticancer Ther. 2014;14:947-54.

17. Hobsley M, Tovey FI, Holton J. Helicobacter pylori and gastric cancer: neither friend nor foe. Gastroenterology. 2007;132:2076.

18. Lin YS, Chen MJ, Shih SC, Bair MJ, Fang CJ, Wang HY. Management of Helicobacter pylori infection after gastric surgery. World J Gastroenterol. 2014;20:5274-82.

19. Lee Y, Tokunaga A, Tajiri T, Masuda G, Okuda T, Fujita I, et al. Inflammation of the gastric remnant after gastrectomy: mucosal erythema is associated with bile reflux and inflammatory cellular infiltration is associated with Helicobacter pylori infection. J Gastroenterol. 2004;39:520-6.

20. Lynch DA, Mapstone NP, Clarke AM, Jackson P, Dixon MF, Quirke P, et al. Cell proliferation in the gastric corpus in Helicobacter pylori associated gastritis and after gastric resection. Gut. 1995;36:351-3.

21. Sakakibara M, Ando T, Ishiguro K, Maeda O, Watanabe O, Hirayama $Y$, et al. Usefulness of Helicobacter pylori eradication for precancerous lesions of the gastric remnant. J Gastroenterol Hepatol. 2014;29 Suppl 4:60-4.

22. Jung DH, Kim JH, Chung HS, Park JC, Shin SK, Lee SK, et al. Helicobacter pylori eradication on the prevention of metachronous lesions after endoscopic resection of gastric neoplasm: a meta-analysis. PLoS One. 2015;10:e0124725.

23. Eom BW, Lee JH, Choi IJ, Kook MC, Nam BH, Ryu KW, et al. Pretreatment risk factors for multiple gastric cancer and missed lesions. J Surg Oncol. 2012;105:813-7.

24. Nasu J, Doi T, Endo H, Nishina T, Hirasaki S, Hyodo I. Characteristics of metachronous multiple early gastric cancers after endoscopic mucosal resection. Endoscopy. 2005;37:990-3.

25. Bair MJ, Wu MS, Chang WH, Shih SC, Wang TE, Chen CJ, et al. Spontaneous clearance of Helicobacter pylori colonization in patients with partial gastrectomy: correlates with operative procedures and duration after operation. J Formos Med Assoc. 2009;108:13-9. 